Conclusions Severe illness and more aggressive management were major risk factors for acute stroke. Traditional vascular risk factors and Caucasian ethnicity were risk factors for ischaemic stroke, while Hispanic or black ethnicity, higher $\mathrm{PaO}_{2}$, and ECMO were significant risk factors for haemorrhagic stroke.

\section{NERVE EXCITABILITY AND MOTOR UNIT NUMBER ESTIMATION: EARLY BIOMARKERS OF NERVE INVOLVEMENT IN HEREDITARY AMYLOIDOSIS (ATTRV)}

1,2,3,4 Antonia Carroll, ${ }^{1}$ Cindy Lin, ${ }^{1}$ Susanna Park, ${ }^{5}$ Neil Simon, ${ }^{2}$ Mary Reilly, ${ }^{3}$ Steve Vucic ${ }^{1}$ Matthew Kiernan. 'Brain and Mind Research Centre, Camperdown, NSW, Australia; ${ }^{2}$ MRC Centre for Neuromuscular diseases, National Hospital of Neurology and Neurosurgery, London, UK; ${ }^{3}$ Westmead Hospital, University of Sydney, Sydney, NSW, Australia; ${ }^{4}$ St Vincent's Hospital, University of NSW, Sydney, NSW, Australia; ${ }^{5}$ Northern Clinical School, University of Sydney, Sydney, NSW, Australia

\subsection{6/bmjno-2021-ANZAN.36}

Objective Gene silencing treatments for hereditary transthyretin amyloidosis (ATTRv) have recently been developed with dramatic improvements observed in patient outcomes. However, the optimal time to initiate treatment is not yet known. The aim of this study is to explore the pathophysiological progression of neuropathic features of ATTRv using nerve excitability and motor unit number estimation.

Methods We prospectively recruited 14 symptomatic patients and 7 asymptomatic carriers and with varied TTR mutations and compared these to 21 healthy controls. Nerve excitability properties of ulnar motor and sensory axons, and ulnar-ADM motor unit number estimation was collected.

Results 'Fanning in' of threshold electrotonus was observed in the motor axons of symptomatic ATTRv patients, suggestive of membrane depolarisation. Motor unit number estimation demonstrated a significant reduction in mean unit number between symptomatic and asymptomatic ATTRv patients ( $p$ $=0.04$ ), with declines seen according to FAP stage and PND score. Significantly increased hyperpolarising current/threshold gradients were seen in sensory axons between symptomatic ATTRv patients and healthy controls $(p=0.002)$, suggesting that upregulation of inwardly rectifying conductance may underlie sensory symptoms and neuropathic pain in ATTRv amyloidosis.

Conclusions These findings suggest that ulnar nerve excitability and motor unit number estimation could be used as a tool to identify early nerve disease in ATTRv and monitor progression.

\section{THE GUT MICROBIOME IN PARKINSON'S DISEASE: LONGITUDINAL INSIGHTS INTO DISEASE PROGRESSION AND THE USE OF DEVICE-ASSISTED THERAPIES}

\footnotetext{
${ }^{1,2}$ Michal Lubomski, ${ }^{3}$ Xiangnan $\mathrm{Xu},{ }^{4}$ Andrew J Holmes, ${ }^{3}$ Samuel Mueller, ${ }^{3}$ Jean Yang, ${ }^{1,2}$ Carolyn M Sue, ${ }^{2}$ Ryan L Davis. 'Neurology Department, Royal North Shore Hospital, St Leonards, NSW, Australia; ${ }^{2}$ Department of Neurogenetics, Kolling Institute, University of Sydney and Royal North Shore Hospital, Sydney, NSW, Australia; ${ }^{3}$ School of Mathematics and Statistics. Sydney Precision Bioinformatics, University of Sydney, Camperdown, NSW, Australia; ${ }^{4}$ School of Life and Environmental Sciences, The Charles Perkins Centre, University of Sydney, Sydney, NSW, Australia
}

10.1136/bmjno-2021-ANZAN.37
Objectives Previous studies have reported altered gut microbiome (GM) composition in association with motor and nonmotor symptoms in Parkinson's disease (PD). Only a few prior studies considered the influences of PD medications, namely oral therapies, on the GM. We investigated the temporal stability of GM profiles from PD patients initiating deviceassisted therapies (DAT) and in a separate cohort characterise GM influences on PD progression.

Methods Clinical data from validated questionnaires and stool samples from 74 PD patients and 74 healthy controls (HCs) were longitudinally evaluated, at $t=0,6$ and 12 months. PD patients were sub-stratified as faster or slower progressors, inferred from levodopa equivalence dose and motor severity measures. Additionally, 19 PD patients receiving Deep Brain Stimulation or levodopa-carbidopa intestinal gel were longitudinally evaluated at $\mathrm{t}=0,6$ and 12 months post-therapy initiation.

Results Persistent underrepresentation of short-chain fatty-acidproducing bacteria, Clostridium XVIII, Butyricicoccus and Fusicatenibacter was apparent in PD patients compared to HCs. No persisting GM profiles were recognised between faster and slower progressing patients, although predictive modelling supported the use of GM profiles to assist in defining PD progression. Our previous findings of acute GM changes in response to DAT initiation were not sustained at 6 and 12 months, although differing microbiota profiles persisted following DAT initiation.

Conclusions We present the largest longitudinal GM study in PD patients showing persistently altered GM profiles indicative of underrepresentation of short-chain fatty-acid-producing bacteria. DAT's were found to exert acute variable influence on the GM that didn't persist over time.

\section{RESOLVING INFECTIOUS MENINGITIS IN UGANDA WITH METAGENOMICS AND HOST TRANSCRIPTOMICS}

1,2Prashanth Ramachandran, ${ }^{1}$ Akshaya Ramesh, ${ }^{3}$ Fiona Creswell, Annie Wapniarski 1 , ${ }^{1}$ Carson Quinn, ${ }^{4} \mathrm{M}$ Rutakinginwa, ${ }^{5}$ Ananta Bandigwala, ${ }^{4} \mathrm{E}$ Kagimu, ${ }^{4} \mathrm{KT}$ Kandole, ${ }^{6}$ Kelsey Zorn, ${ }^{4} \mathrm{~L}$ Tugume, ${ }^{4} \mathrm{~J}$ Kasibante, ${ }^{4} \mathrm{~K}$ Ssebambulidde, ${ }^{4} \mathrm{M}$ Okirwoth, ${ }^{5} \mathrm{Nathan}$ Bahr, ${ }^{4}$ Abdu Musubire, ${ }^{6}$ Amy Lyden, ${ }^{7}$ Paula Serpa, ${ }^{7}$ Gloria Castaneda, ${ }^{7}$ Saharai Caldera, ${ }^{7}$ Chaz Langelier, ${ }^{7}$ Emily Crawford, ${ }^{5}$ David Boulware, ${ }^{5}$ David Meya, ${ }^{1}$ Michael Wilson. ${ }^{1}$ Weill Institute for Neurosciences, University of California, San Francisco, San Francisco, California, USA; ${ }^{2}$ University of Melbourne, Melbourne, VIC, Australia; ${ }^{3}$ Clinical Research Department, London School of Hygiene and Tropical Medicine, London, WC1E 7HT, UK; ${ }^{4}$ Infectious Diseases Institute, Makerere University, Kampala, Uganda: ${ }^{5}$ University of Minnesota, Minneapolis, Minnesota, USA; ${ }^{6}$ University of California San Francisco, San Francisco, CA USA; ${ }^{7}$ Chan Zuckerburg Biohub, San Francisco, California, USA

\subsection{6/bmjno-2021-ANZAN.38}

Objectives Tuberculous meningitis(TBM) is a common cause of meningitis in sub-Saharan Africa. CSF PCR with GeneXpert RIF/MTB Ultra is only 70\% sensitive for detection of definite/ probable TBM. Many infections can mimic TBM. Metagenomic next generation sequencing(mNGS) can detect the whole diversity of infectious microbes, but can be insensitive to TB in CSF. We assessed whether leveraging CSF mNGS to identify infections combined with a machine learning classifier (MLC), based on host transcriptomic data generated by mNGS, could enhance diagnostic accuracy for TBM.

Methods Prospectively enrolled 347 HIV-infected Ugandan adults with subacute meningitis: RNA/DNA libraries were made from CSF and deep sequenced. Non-human sequences 
were interrogated to identify pathogens. A host transcriptomic MLC was developed from human RNA transcripts using 70 cases. The MLC and mNGS reporting thresholds were then tested on 108 blinded cases within the cohort.

Results mNGS was $75 \%$ concordant(27/36) for detecting TB in definite TBM cases and 59\% concordant(30/51) in definite/ probable TBM combined. $3 \mathrm{~TB}$ and 3 non-TB pathogens were detected in the probable TBM group. In the possible TBM/ indeterminant groups, mNGS identified 3 cases of TBM and 17 other pathogens. The combined mNGS and host-MLC displayed $83.3 \%(5 / 6)$ sensitivity, $86.8 \%(59 / 68)$ specificity, with an area under the ROC curve of $0.83(\mathrm{p}=0.009)$.

Conclusion mNGS identified an array of infectious TBM mimics, including many treatable and vaccine preventable pathogens. mNGS was $75 \%$ concordant with definite TBM. We further enhanced the sensitivity of the CSF mNGS assay by developing the first CSF-based host MLC to discriminate between TBM and its mimics

\section{PREVALENCE OF MRI SIGNS OF INTRACRANIAL HYPERTENSION AND THEIR ASSOCIATION WITH PAPILLEDEMA: A PROSPECTIVE STUDY USING OCULAR FUNDUS PHOTOGRAPHY}

\footnotetext{
1,2,3Benson S Chen, 'Benjamin I Meyer, 'Amit M Saindane, 'Beau B Bruce, 'Nancy J Newman, 'Valérie Biousse. 'Emory University School of Medicine, Atlanta, GA, USA; ${ }^{2}$ Department of Clinical Neurosciences, University of Cambridge, Cambridge, UK; ${ }^{3}$ Cambridge Eye Unit, Addenbrooke's Hospital, Cambridge, UK
}

\subsection{6/bmino-2021-ANZAN.39}

Objectives MRI signs of intracranial hypertension (MRI-IH) are classically associated with idiopathic intracranial hypertension (IIH), but also detected in asymptomatic individuals without papilledema. The objective of this study was to determine the prevalence of MRI-IH in consecutive outpatients undergoing brain MRI for any clinical indication, and explore their association with papilledema.

Methods Prospective cross-sectional observational study of outpatients undergoing brain MRI, with ocular fundus photographs taken concurrently. Radiologic studies were analyzed for MRI-IH. Univariate analysis with Fisher's exact test or ttest was performed.

Results Of 296 patients included, the most common indication for MRI was surveillance of a brain neoplasm (27.7\%). Investigation of headaches $(8.8 \%)$ or disorders of raised ICP $(1.4 \%)$ were uncommon. At least one MRI-IH was present in $49 \%$ of patients [empty sella (33.1\%), enlarged Meckel's cave $(15.9 \%)$, increased peri-optic CSF $(10.8 \%)$, optic nerve tortuosity (7.8\%), scleral flattening $(0.7 \%)$, cephaloceles $(1.4 \%)$. Bilateral transverse venous sinus stenosis (TVSS) was present in $3.0 \%$ of 198 patients. Five patients $(1.7 \%)$ had papilledema. Compared to patients without papilledema, those with papilledema had significantly higher BMI and prior history of IIH, and increased prevalence of empty sella, optic nerve tortuosity, and TVSS on MRI. The prevalence of papilledema increased from $2.8 \%$ among patients with at least one MRI-IH to $40 \%$ among patients with four or more MRI-IH.

Conclusion MRI-IH are common in patients undergoing brain MRI, but rarely associated with papilledema. The management of patients with incidentally detected MRI-IH likely does not require systematic lumbar puncture unless concerning symptoms or papilledema are present.

\section{THE QUEST TO REDUCE STROKE TREATMENT DELAYS AT A MELBOURNE METROPOLITAN PRIMARY STROKE CENTRE OVER THE LAST TWO DECADES}

1,2 2 eter SW Park, ${ }^{1}$ Tanya Frost, ${ }^{1}$ Peter SY Tan, ${ }^{1}$ Joseph Wong, ${ }^{2}$ Alun Pope, ${ }^{1,2}$ Helen M Dewey, ${ }^{1,2}$ Philip MC Choi. 'Department of Neurosciences, Box Hill Hospital, Eastern Health, Box Hill, VIC, Australia; ' 2 Eastern Health Clinical School, Faculty of Medicine, Nursing and Health Sciences, Box Hill, VIC, Australia

\subsection{6/bmino-2021-ANZAN.40}

Objectives To examine door-to-needle time (DNT) trends and the impact of continuous quality improvement initiatives since thrombolysis became standard of care in Australian metropolitan setting for eligible acute ischaemic stroke patients.

Methods Single-centre retrospective cohort study of consecutive patients treated with thrombolysis at high volume primary stroke centre from January 2003 to December 2019. Primary outcomes are DNT, and annual number of 'Code Stroke' activations and stroke admissions. Regression modelling for potential factors associated with DNT.

Results 1,250 patients were treated with thrombolysis over 17 years; 54\% were male with a median age of 76 (interquartile ranges [IQR], 66-83). Median DNT fluctuated between 70 to 93 minutes (IQR, 55-95 to 82-120) from 2003 to 2012, reaching 60 minutes in 2013 and nadir of 47 minutes in 2014. Median DNT then decreased from 58 minutes in 2015 to 51 minutes in 2019 with progressive tightening of IQR (46-78 to 40-62). Number of patients treated within 60 minutes of hospital arrival was less than $30 \%$ between 2003-2012. This rose to an average of $63 \%$ during 2015-2018 and 71\% in 2019. From 2015 to 2019, per annum number of 'Code Stroke' activations increased from 940 to 1300 while stroke admissions plateaued at 750 . 'Direct-to-CT' protocol and acute stroke presence were two modifiable workflow factors independently associated with faster DNT $(\mathrm{P}<0.001)$.

Conclusion Targeted quality improvement initiatives are key to reducing treatment delays in the Australian metropolitan setting. Relative stagnation in DNT improvement is concerning and needs further investigation.

\section{COGNITIVE IMPAIRMENT IN LATE ONSET EPILEPSY}

Xin Zhang, Armin Nikpour, Rebekah Ahmed. Royal Prince Alfred Hospital, Camperdown, NSW, Australia

\subsection{6/bmjno-2021-ANZAN.4}

Objective Late onset, unprovoked epilepsy patients with cognitive impairment can have complex pathophysiology. ${ }^{1}$ Our objective was to study the characteristics and contributors of cognitive impairment in this group; and how patients with dementia could be differentiated from late onset epilepsy patients.

Methods Twenty-six patients with epilepsy, onset after 50 years of age, with new cognitive complaints and 26 patients with clinically diagnosed Alzheimer's Disease (AD) were recruited. These participants had comprehensive neuropsychological and neuroimaging assessments. A subset of 17 participants from the Epilepsy group underwent longitudinal neuropsychological assessment.

Results In the Epilepsy group, the neuropsychological profile of cognitive impairment was consistent with the foci and severity of seizure activity in $46 \%$ of participants; subcortical 